\title{
Early Intervention in Mood and Anxiety Disorders: The First Episode Mood and Anxiety Program (FEMAP)
}

Elizabeth A. Osuch, Evelyn Vingilis, Sandra Fisman and Carolyn Summerhurst

\begin{abstract}
The First Episode Mood and Anxiety Program (FEMAP) was developed for youth with mood and/or anxiety concerns in London, Ontario, to provide early intervention for these disorders, similar to the first-episode psychosis programs across Ontario and Canada. The logic and causal models of the pathway to and through FEMAP are described and inclusion/exclusion criteria are delineated. Results of the process evaluation of the model and preliminary data from a treatment-effectiveness evaluation and ongoing costcomparison evaluation are provided. Several characteristic quotes from youth utilizing the service are included, as is an overview of utilization statistics. Challenges and lessons learned are conveyed.
\end{abstract}

\section{Background}

Many facts support early intervention. First, $75 \%$ of all mental illnesses start before the age of 25 (Kessler et al. 2007). Second, early intervention is the medical standard for chronic and/ or episodic illnesses, including diabetes (Nathan et al. 2007), chronic pain (MTUS 2009), heart disease (Grady et al. 2000), arthritis (Emery 2002), multiple sclerosis (Jacobs et al. 2000) and cancer (Smith et al. 2006). Third, provincial and national initiatives for early identification and intervention of psychosis have been standard for over a decade (Ehmann et al. 2004). Finally, mood and anxiety disorders respond well to treatment, particularly if treated early (Perry et al. 1999; Kennard et al. 2009; Rapee et al. 2009; Berk et al. 2011; Otte 2011).

Given these facts, it is remarkable that, in 2006, no services were available for psychiatric help for teenagers with a mood or anxiety problem in London, Ontario, with a wait time of less than 18 to 24 months. Moreover, accessible psychiatric care was ridiculously brief - one to two visits to the hospital's urgent consultation service, usually accessed through the emergency room (ER) or other physician referral. The expense to the system determined by this care pathway, almost necessitating a visit to the ER, was equally untenable. And this situation was not unique to London, Ontario (EAO, personal communication).

The negative economic consequences of non-treatment of mental illnesses far exceed the direct costs of treatment $(\mathrm{Hu}$ 2006). For mood and anxiety disorders, the hidden cost of non-treatment may include youth dropping out of school, thereby not achieving their academic potential, which in turn leads to underemployment and the need for social assistance. Disturbed family and friend relationships are also common and, without intervention, there is further deterioration of youth's social supports. Some youth resort to substance abuse to manage their symptoms. Suicide, the second leading cause of death in 15- to 24-year-olds in Canada (Langlois and Morrison 2002), can be the final conclusion to untreated mood and anxiety disorders. 
The Rea Chair of Affective and Anxiety Disorders was created through a generous donation from the Rea Family Foundation. Filled in late 2005, the position established a commitment to furthering research excellence in the understanding and treatment of mood and anxiety disorders. The incoming psychiatrist $(\mathrm{EAO})$ was interested in brain imaging and the neurocircuitry of reward processing in major depressive disorder in the early stages, before patients have lived through years of debilitating illness. This led to the emphasis on identifying late teens and young adults with depression in 2006. What was not a surprise was that depression in youth was prevalent. What was a surprise was that after completing research protocols investigating brain pathophysiology there was nowhere to send these youth for treatment. That was the inspiration for the First Episode Mood and Anxiety Program (FEMAP).

\section{Local Innovation}

FEMAP started as one psychiatrist (EAO) with the ardent support of the then department chair (SF). FEMAP was modelled on the early psychosis program in London - the Psychosis and Early Intervention Program for Psychoses (PEPP) (Norman and Manchanda 2015). PEPP includes the ability to self-refer, emphasis on ambulatory services, short wait times, no artificial discharge dates and focus on first episode. In addition, the integration of clinical care and clinical research is standard.

With that backdrop, FEMAP took shape. Mood and anxiety disorders caught early are treatable with psychotherapy and psychopharmacology, even though they often recur (Perry et al. 1999; Morriss et al. 2007; March et al. 2009; Rapee et al. 2009). By 2011, funded by the department of psychiatry, charitable donations and research project funding, FEMAP had 1.6 fulltime equivalents of psychiatrist, a full-time intake assessment clinical social worker, a one-day-per-week addictions therapist, a one-day-per-week family therapist and a receptionist. By 2013, charitable donations had supported hiring a psychologist two days per week.

In early 2009, with the help of the department chair (SF) and support of the St. Joseph's Health Care Hospital board, FEMAP moved from limited hospital space to a renovated house in the community. This provided a much friendlier, welcoming and less stigmatizing environment for youth. With guidance from our health systems evaluation expert (EV), we set about to fully describe and evaluate FEMAP.

FEMAP's goal is to catch mood/anxiety disorders early, identify youth and treat them effectively before they veer off their normal developmental trajectory. The causal and logic models, updated from previous versions (Ross et al. 2012; Osuch et al. 2015), are illustrated in Figure 1. The upper diagram reflects experiences/changes expected in youth with $\mathrm{mood} /$ anxiety problems on their pathway to and through care; the logic model presents program activities to effect the expected changes. The logic model includes outputs (program activities) and short-, medium- and long-term outcomes, such as a reduction in ER and inpatient psychiatric service utilization, school dropouts and suicide attempts.

Programmatic activities of Phase I involve community outreach, in-person and in-depth assessment and the establishment and communication of recommendations to youth. The recommendations include acceptance into FEMAP for treatment versus the decision that the youth would be better served in another program or, in rare cases, reassured that no specialty mental healthcare is needed. If youth are referred elsewhere, facilitation of that transition is attempted.

Phase II involves full psychiatric or psychological evaluation, treatment and recovery from illness. Two possible long-term outcomes are: 1) sustained recovery from illness or 2) eventual recurrence of illness requiring episodic care or lack of remission and the need for prolonged care. Due to the natural course of mood and anxiety disorders, some youth will have sustained recovery while others will experience repeated episodes of illness or chronic illness.

Adherence to strict inclusion/exclusion criteria has been essential to optimize scarce resource utilization. These criteria for FEMAP are as follows: mood or anxiety symptoms as the primary problem; age 16-25 (previously 16-26); no developmental delay or severe learning disability; no primary substance use that preceded the onset of mood/anxiety symptoms; no major medical problems; no ongoing legal charges; and less than 18 months of prior psychiatric medication treatment. These criteria were designed for one primary purpose - maximizing the probability that youth admitted into FEMAP could recover with the level of treatment available, given the lack of resources. Youth were screened by using a brief telephone interview.

\section{Results of Innovation}

\section{Pathway to Care}

Phase I has undergone a process evaluation to determine whether it has been meeting its goals as a pathway to care; the results indicate that the outreach was effective and the model was attracting youth in need of specialty psychiatric care, as indicated by their severity of symptoms and level of functional impairment (Ross et al. 2012; Osuch et al. 2015). Given the ability of youth to access FEMAP without a physician referral, careful screening according to FEMAP inclusion/exclusion criteria and a full in-person assessment by a skilled clinician have been important in reducing over-inclusion of inappropriate patients, a potential negative unintended consequence of open access.

In the early evaluation, approximately $73 \%$ of 548 youth who contacted FEMAP were appropriate and were accepted into the program after screening and assessment (Osuch et al. 


\section{FIGURE 1.}

Pathway through care and Logic model of the First Episode Mood and Anxiety Program (FEMAP).

A. Pathway through care of FEMAP, including two phases that support youth on their trajectory through care. Phase I consists of outreach, intake and assessment of youth with mood/anxiety concerns. Phase II involves full psychiatric/psychological evaluation, and then treatment and, ideally, recovery to baseline functioning.

\section{Brief telephone sxreen}

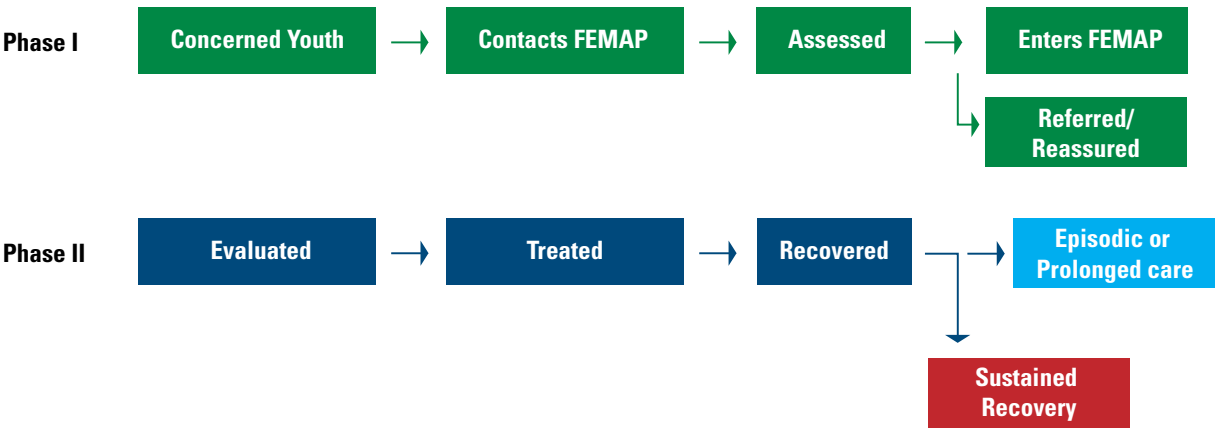

B. Logic model indicates outputs (activities and participation) as well as outcomes or impact, including short-, medium- and long-term impacts. Potential unintended consequences are listed as well. FMD = family medical doctor.

\begin{tabular}{|c|c|c|c|c|}
\hline \multicolumn{2}{|c|}{ Outputs } & \multicolumn{3}{|c|}{ Outcomes - Impact } \\
\hline Activities & Participation & Short-term & Medium-term & Long-term \\
\hline $\begin{array}{l}\text { Phase I: } \\
\text { Intake process allows for } \\
\text { self-referral } \\
\text { Assess and identify: level of } \\
\text { symptom severity; symptom } \\
\text { clusters; level of functional } \\
\text { impairment } \\
\text { Direct youth to needed } \\
\text { services: FEMAP or other }\end{array}$ & $\begin{array}{l}\text { Youth age } 16-25 \text { with concerns } \\
\text { related to mood and/or anxiety } \\
\text { with or without substance use } \\
\text { FEMAP staff provide assess- } \\
\text { ment of youth with mental } \\
\text { health and substance use } \\
\text { concerns } \\
\text { Psychiatric, psychological, } \\
\text { addictions and family therapy } \\
\text { specialty treatment services in }\end{array}$ & $\begin{array}{l}\text { Direct access of youth } \\
\text { with significant mood/ } \\
\text { anxiety concerns to mental } \\
\text { health services, when } \\
\text { they're ready } \\
\text { Less usage of Emergency } \\
\text { Services as first point of } \\
\text { contact for psychiatric } \\
\text { care by youth }\end{array}$ & $\begin{array}{l}\text { Reduction in functional } \\
\text { impairment of youth with } \\
\text { mood/anxiety concerns } \\
\text { Recovery from mood/ } \\
\text { anxiety and substance } \\
\text { use disorder(s) }\end{array}$ & $\begin{array}{l}\text { Fewer untreated youth } \\
\text { with mental illness } \\
\text { Reduced youth school drop- } \\
\text { out and unemployment } \\
\text { Reduced youth suicide } \\
\text { Reduced inpatient } \\
\text { psychiatric service use } \\
\text { Reduced stigma; faster } \\
\text { recovery }\end{array}$ \\
\hline $\begin{array}{l}\text { Phase II: } \\
\text { Provide treatment, as } \\
\text { clinically indicated }\end{array}$ & one locatıon & $\begin{array}{l}\text { Potential Unintended Con } \\
\text { Excessive use of psychiatric } \\
\text { such services-cost implica } \\
\text { With no FMD referral, psych }\end{array}$ & $\begin{array}{l}\text { quences: } \\
\text { ecialty services by youth wh } \\
\text { trist is left doing long-term c }\end{array}$ & are not ill enough to warrant \\
\hline
\end{tabular}

2015). Recent, newer data indicate that this proportion has increased to $85 \%$ in a sample of 607 youth calling for services or referred by a physician, suggesting that the community is getting better at self- (and physician-) screening. Of 897 youth recently assessed, less than $5 \%$ were not accepted but only reassured that no specialty services were needed. This indicated that over-inclusion was minimal (Osuch et al. 2015) and the FEMAP telephone screening criteria were highly effective at minimizing clinician time spent with youth not needing specialty care.

The pathway evaluation also revealed that the term first episode is a misnomer in that between 60 and $70 \%$ of youth assessed at FEMAP had received prior treatment for mood and/ or anxiety concerns (Osuch et al. 2015). This was also evident based on the clinical history of many youth who had not sought prior professional help, but first episode was retained in the program title to encourage youth to connect even when they are unsure if they are "sick enough" to justify reaching out for help.

Referral sources for accessing the program varied. Between 2009 and 2015, a majority of youth (63\% of 1332) who came to FEMAP did so from non-physician referrals. In recent years, non-physician referrals included those from post-secondary school services such as university or college guidance (11\%) and secondary school services (5\%). 


\section{Treatment Effectiveness and Cost Comparison}

Phase II involves full psychiatric/psychological evaluation, treatment and recovery back to baseline functioning (Figure 1A). The goal of treatment is full recovery with no artificial termination of treatment based on time. Treatment goals include stabilization so that specialty mental healthcare is no longer required; medication administration can be taken over by a family doctor (FMD), if necessary and available.

Figure 2 illustrates the course of symptom severity for depression and anxiety in the first 88 FEMAP patients to be evaluated in our ongoing longitudinal, prospective treatment-effectiveness study. It also illustrates functional improvement and life satisfaction for the same time period. Each clinical measure indicated highly statistically significant improvement over the course of treatment. Further results are pending completion of this effectiveness study. The measure shown in Figure 2A is the Montgomery-Asberg Depression Rating Scale (MADRS) self-report version, which has 9 questions, and possible scores range from 0 to 27; high score indicates low $\mathrm{mood} /$ higher depression and scores greater than 10 indicate probable or undoubted need for treatment (shown by the horizontal line in Figure 2A). MADRS scores were highly significantly better at 4-month follow-up, and fell below the threshold score for need for treatment. The Reiss-Epstein-Gursky Anxiety Sensitivity Index (ASI) (Figure 2B) has 16 questions with possible scores ranging from 0 to 64; high score indicates greater anxiety. The Sheehan Disability Scale (SDS)(Figure 2C) has three Likertscale questions resulting in a total score ranging from 0 to 30 . A score of 5 on any one of the subscales indicates clinically significant functional impairment. The bar graph illustrates mean total scores at the three time points shown; there was highly significant improvement in functioning at the 4-month follow-up. The Quality of Life Enjoyment and Satisfaction Questionnaire - Short Form (QLESQ)(Figure 2D) has 14-item scale ranging from 14 to 70 . Again, the bar graph shows that the youth in treatment at FEMAP experienced a highly significant improvement in their life enjoyment and satisfaction scores.

In spite of high rates of remission in youth with mood/ anxiety disorders, there are several possible long-term outcomes. Recovery can be sustained over time or the course of illness can be marked by recurrent episodes, or, for the most ill patients, by the need for prolonged care and incomplete recovery. The probability of subsequent depressive episodes is significantly higher after one depressive episode, even when treatment is optimal (Melartin et al. 2004; Vittengl et al. 2007). Anxiety disorders also can recur (Nay et al. 2013). Thus, as with other episodic illnesses,

FIGURE 2.

Treatment effectiveness of the FEMAP model for the first 88 patients evaluated in phase II of the pathway through care

A.

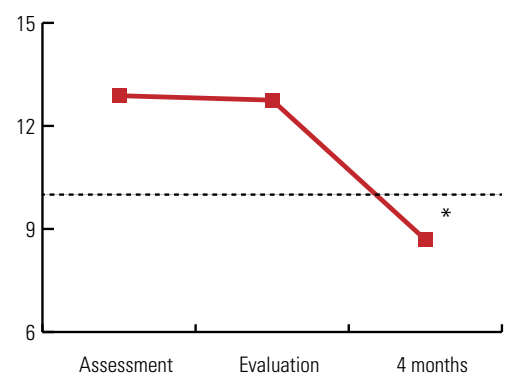

B.

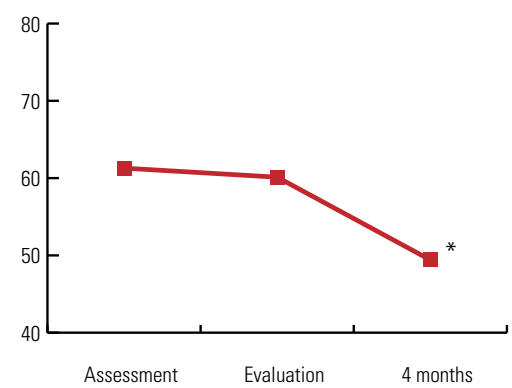

C.

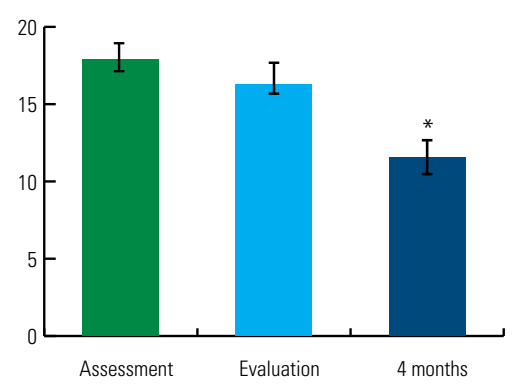

D.

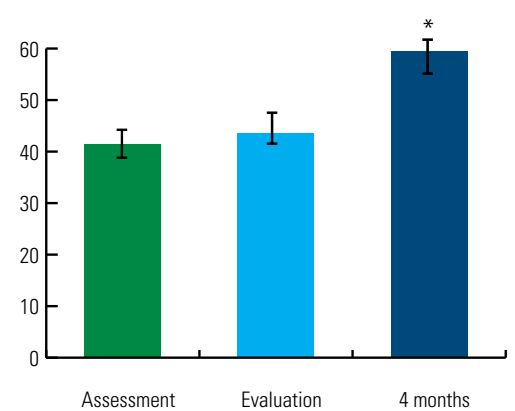

* indicates clinically significant changes of $p<.001$ at time points indicated, as assessed with repeated measures ANOVA. 
establishing a positive working relationship with care providers is important so that repeat episodes will be captured early and longterm problems prevented. Often, the only difference between a mild-to-moderate mental illness and a chronic and persistent one is the passage of time and the absence of adequate treatment. A long-term, one-plus year follow-up study of the therapeutic effectiveness and cost-effectiveness of FEMAP is underway.

FEMAP allows youth to return to treatment for subsequent episodes of care, provided they have not drastically aged out of the program. When a youth has been highly engaged with the program, gotten better and been discharged, only to have a recurrence several years later, it is clearly most clinically and economically efficient to have FEMAP treat that individual again.

Formal cost effectiveness studies of FEMAP are pending, but data using several relevant cost comparisons have proven illustrative. In the United Kingdom, the London School of Economics found that paying for non-physician mental healthcare providers (social workers, psychologists) to conduct treatment through the National Health Service provided an overwhelming savings compared with the cost of social assistance (Layard 2006). To conduct a similar but simpler analysis, we compared the cost of 4 months of treatment at FEMAP to several other common mental healthcare-related costs.

The average cost of 4 months of treatment at FEMAP for the first 63 patients in our study was determined by chart review. These costs included all clinician sessions (psychiatrist, psychologist, addictions worker; all group or individual therapy sessions), laboratory tests ordered, and medications prescribed by FEMAP clinicians. This averaged $\$ 1634$ per patient. In contrast, the cost of compelling an individual to a psychiatric evaluation in an ER using Form 1 of the Mental Health Act in Ontario, plus the cost of the ER visit, was approximately $\$ 2188$ per patient, based on 330 such cases at the London Health Sciences Centre in fiscal year 2013-2014. Further, the cost of being on the Ontario Disability Support Program for 4 months, based on the pay-out costs to the provincial budget as of September/October of 2014, was $\$ 4392$ per patient. From these estimated cost comparisons, it is reasonable to suspect that effective, early, outpatient treatment of youth with mood/ anxiety disorders may be a much wiser investment of social dollars than failing to treat these illnesses in the early stages and paying the longer-term consequences.

\section{Patient Experience}

In the context of a research project, we interviewed patients about their past experiences with mental healthcare in general and with FEMAP in particular. Responses revealed how youth experienced the mental healthcare system and the path to receiving care within it. Illustrative quotes are below.

Comparing previous mental healthcare services to FEMAP, one young man stated: ...outside of FEMAP, trying to find an appointment with a psychiatrist was freaking ludicrous, like it was unreal, and I can't even begin to imagine the situation I would have been in if I wasn't supported by my parents. Like I don't even know, man, what people must be going through. It was insane. So that like blew my mind, especially when we talk about how this is Canada and universal healthcare and all that, and it's completely inaccessible. (male, age 25)

About getting help at FEMAP: "It has gotten easier. The first couple of times... it's the hardest thing in the world trying to open up to a complete stranger, [to tell them] something you've never told another living soul."

About his treatment provider at FEMAP this individual stated:

She really does seem to care, and uhh, [is] talking a lot more than any other doctor I've had. Like I was afraid of it at first, but I've really started to - I don't think 'enjoy' is the right word - but it's good... This is the first place I've come to where I've actually felt a proper patient-doctor dynamic. I'm more than just a chart. They actually want to find out what's wrong and deal with that.

In contrast, regarding prior treatment in other settings, he stated: "And, yeah, just the in and out, the in and out like, 'Just take these pills,' just doesn't work." (male, age 26)

There is an expectation that FMDs will be the major providers of mental healthcare for youth with mood and anxiety concerns. However, some youth had strong opinions about that model:

I was uncomfortable especially because a family doctor to me doesn't ... like you go there for physical things. That's what was in my mind and I was like, 'How am I supposed to tell him what's going on? Like, he's probably going to judge me and stuff like that, because I'll have to go back to him later to talk about physical things and it might be awkward'.... It was very awkward, yeah. Umm, he is definitely more of, like, a medical kind of guy and I didn't feel comfortable telling him my feelings at all and umm, I just wanted to get out of there as soon as possible. (female, age 18)

The need to have an empathic treatment provider who cared emerged repeatedly.

Well, I was treated like a person, not a ... just some experiment or study. I sort of expected having [the treatment providers at FEMAP] just be there to help me get better, but I didn't think that they would necessarily invest all the 
time and emotion that they did into me.... But they still did because they genuinely cared about me, and I wasn't expecting that. (female, age 17)

\section{Challenges and Ongoing Need}

There have been challenges along the way. FEMAP has assumed care for several patient groups that it was not specifically designed for. This was done to provide care for youth who would have otherwise gone untreated. This has included youth with posttraumatic stress disorder; youth with eating disorders (ED) not ready to accept specialized ED treatment but who had substantial mood/anxiety symptomatology; and youth with substance use disorders, mostly isolated to cannabis and/ or alcohol use. Secondary substance use is quite common in youth presenting with mood/anxiety symptoms and we have found cannabis use to be associated with lower functioning in this population (Osuch et al. 2013).

Conversely, a feature that FEMAP intended to maintain but has not been able to is rapid response. There was a $65 \%$ increase in requests for service between March 2013 and May 2015, with over 35 new contacts per month, over $80 \%$ of whom are appropriate for the service. With no resources forthcoming for stable clinical salaries and no way to hire new clinical staff, this has led to long wait times. The wait for entry into FEMAP has gone from 2 weeks to over five months - a wait that is truly unacceptable for an early intervention program. This should be rectified without compromising the quality of service delivered by the program, and the most effective way to do so would be to add psychiatric support.

The complementary challenge is the discharge of youth from the program, as specified as an unintended consequence in the logic model (Figure 1B). While the average youth is substantially improved in only 4 months of treatment or less, some need more. Generally, this is not a matter of determining who will prescribe stable doses of medications to youth who are doing well. Even without a FMD, such youth are not a substantial expenditure to the system because renewing a prescription once or twice per year is not an onerous cost. The problem arises when youth do not get better quickly with treatment.

While some mental healthcare systems address the challenge of treatment refractoriness by placing limits on duration of treatment, such an approach is not consistent with the medical model or best practice (McIntyre and O'Donovan 2004). It would not be considered acceptable for the treatment of other episodic/chronic medical illnesses to terminate treatment based on a certain number of sessions. Consider a clinic for diabetes where people with unstable diabetes are discharged to no care after 10 sessions even when their blood sugar is poorly controlled. Oddly, mental healthcare appears to be unique within the medical model in deciding on end of treatment based on factors other than the patient's condition.

\section{Lessons Learned}

- FEMAP provides an example of a well-characterized healthcare delivery model for early intervention for mood and anxiety disorders in transition-aged youth. The model's pathway to care, treatment effectiveness and cost-effectiveness have all been studied or are under research investigation.

- The program has been able to engage the population it aims to help - youth in the early stages of dysfunction from mood and/or anxiety disorders, with or without secondary addiction issues. Symptom severity and level of function of the youth studied were highly significantly improved with treatment. Youth appear to have a positive experience with the service and feel comfortable with the clinicians they receive care from.

- Self-referral did not result in the over-inclusion of inappropriate patients and appeared to make little difference when discharging youth who no longer needed psychiatric expertise.

- Some youth with other related mental health challenges found help at FEMAP, where alternatives were not otherwise accessible in the community.

- A preliminary cost comparison of FEMAP appeared excellent - the cost of treating mood/anxiety disorders effectively in their early stages is far less than the cost of waiting until youth require ER visits, hospitalization or social assistance. Mood and anxiety disorders with or without comorbid addiction issues account for a large component of mental illnesses with their onset in youth and are highly treatable, making programs such as FEMAP a clear benefit to the overall mental healthcare system design. We conclude that this is an appropriate use of specialty psychiatric resources.

- Provincial commitment to fund programs like FEMAP is imperative. With a wait time now growing from two weeks several years ago to over five months currently, FEMAP is destined to collapse as it fails to meet the rapid response needed for an early intervention strategy, even while the need/demand for its services increases.

- Next steps could include incorporation of this model into a test site of province-wide hubs to provide comprehensive care to transition-aged youth, as inspired by the Boeckh Foundation and related advocates for youth mental healthcare (Snowdon 2014). 
On the contrary, as long as a young person is continuing to make even small progress and there is no better, alternative place for the youth to get treatment, she or he can continue at FEMAP. Therefore, FEMAP clinicians have had to become sensitive to when youth are not motivated to change and create incentives for them to do so or abandon efforts at treatment and discharge them until such motivation is forthcoming. HQ

\section{Acknowledgements}

The authors would like to thank the FEMAP team during the time of the project. Results of this work were also presented at the Innovation Fund (AMOSO) Showcase; Toronto, ON; 28 November 2013 poster presentation and the Innovation Fund (AMOSO) Showcase; Toronto, ON; 18 November 2010 poster presentation.

\section{Funding and Conflict of Interests}

Dr. Osuch holds an investigator initiated award from Pfizer. There is no conflict of interest with that award and any contents of this manuscript. No conflict of interest exists for this study for any of the authors. This project was funded by grants from the Academic Health Services Centres Alternate Funding Plan Innovation Fund. Support also came from the Schulich School of Medicine and Dentistry, University of Western Ontario, London Health Sciences Centre, the St. Joseph's Health Care System, and the Lawson Health Research Institute.

\section{References}

Berk, M., A. Brnabic, S. Dodd, K. Kelin, M. Tohen, G.S. Malhi, L. Berk, P. Conus and P.D. McGorry. 2011. "Does Stage of Illness Impact Treatment Response in Bipolar Disorder? Empirical Treatment Data and Their Implication for the Staging Model and Early Intervention." Bipolar Disord 13(1): 87-98.

Ehmann, T., M. Gilbert and L. Hanson. 2004. Early Psychosis Intervention: A Framework for Strategic Planning. Vancouver, B.C.: Canadian Mental Health Association.

Emery, P. 2002. "Evidence Supporting the Benefit of Early Intervention in Rheumatoid Arthritis." J Rheumatol Suppl 66: 3-8.

Grady, K.L., K. Dracup, G. Kennedy, D.K. Moser, M. Piano, L.W. Stevenson and J.B. Young. 2000. "Team Management of Patients With Heart Failure: A Statement for Healthcare Professionals From The Cardiovascular Nursing Council of the American Heart Association." Circulation 102(19): 2443-56.

Hu, T.W. 2006. "Perspectives: An International Review of the National Cost Estimates of Mental Illness, 1990-2003." J Ment Health Policy Econ 9(1): 3-13.

Jacobs, L.D., R.W. Beck, J.H. Simon, R.P. Kinkel, C.M. Brownscheidle, T.J. Murray, N.A. Simonian, P.J. Slasor and A.W. Sandrock. 2000. "Intramuscular Interferon Beta-1a Therapy Initiated During a First Demyelinating Event in Multiple Sclerosis. CHAMPS Study Group." N Engl J Med 343(13): 898-904.
Kennard, B.D., S.G. Silva, S. Tonev, P. Rohde, J.L. Hughes, B. Vitiello, C.J. Kratochvil, J.F. Curry, G.J. Emslie, M. Reinecke and J. March. 2009. "Remission and Recovery in the Treatment for Adolescents with Depression Study (TADS): Acute and Long-Term Outcomes." J Am Acad Child Adolesc Psychiatry 48(2): 186-95.

Kessler, R.C., G.P. Amminger, S. Aguilar-Gaxiola, J. Alonso, S. Lee and T.B. Ustun. 2007. "Age of Onset of Mental Disorders: A Review of Recent Literature." Curr Opin Psychiatry 20(4): 359-64.

Langlois, S. and P. Morrison. 2002. "Suicide Deaths and Suicide Attempts." Health Rep 13(2): 9-22.

Layard, R. 2006. “The Case for Psychological Treatment Centres.” Bmj 332(7548): 1030-32.

March, J., S. Silva, J. Curry, K. Wells, J. Fairbank, B. Burns et al. 2009. "The Treatment for Adolescents With Depression Study (TADS): Outcomes Over 1 Year of Naturalistic Follow-Up." Am J Psychiatry 166(10): 1141-49.

McIntyre, R.S. and C. O'Donovan. 2004. "The Human Cost of Not Achieving Full Remission in Depression." Can J Psychiatry 49(3 Suppl 1): 10S-16S.

Melartin, T.K., H.J. Rytsala, U.S. Leskela, P.S. Lestela-Mielonen, T.P. Sokero and E.T. Isometsa. 2004. "Severity and Comorbidity Predict Episode Duration and Recurrence of DSM-IV Major Depressive Disorder." J Clin Psychiatry 65(6): 810-19.

Morriss, R.K., M.A. Faizal, A.P. Jones, P.R. Williamson, C. Bolton and J.P. McCarthy. 2007. "Interventions for Helping People Recognise Early Signs of Recurrence in Bipolar Disorder." Cochrane Database Syst $\operatorname{Rev}(1):$ CD004854.

MTUS. 2009. "Chronic Pain Medical Treatment Guidelines." Retrieved November 11, 2015. <http://www.dir.ca.gov/dwc/ DWCPropRegs/MTUS_Regulations/MTUS_ChronicPainMedical TreatmentGuidelines.pd $\bar{f}>$.

Nathan, D.M., M.B. Davidson, R.A. DeFronzo, R.J. Heine, R.R. Henry, R. Pratley and B. Zinman. 2007. "Impaired Fasting glucose and Impaired Glucose Tolerance: Implications for Care." Diabetes Care 30(3): 753-59.

Nay, W., R. Brown and R. Roberson-Nay. 2013. "Longitudinal Course of Panic Disorder With and Without Agoraphobia Using the National Epidemiologic Survey on Alcohol and Related Conditions (NESARC)." Psychiatry Res 208(1): 54-61.

Norman, R.M.G. and R. Manchanda. 2016. "Prevention and Early Intervention Program for Psychoses (PEPP).” Healthcare Quarterly 18 (Special): 37-41. doi:10.12927/hcq.2016.24442

Osuch, E., E. Vingilis, E. Ross, C. Forster and C. Summerhurst. 2013. "Cannabis Use, Addiction Risk and Functional Impairment in Youth Seeking Treatment for Primary Mood or Anxiety Concerns." International Journal of Adolescent Medicine and Health 25(3).

Osuch, E.A., E. Vingilis, C. Summerhurst, C.I. Forster, E.E. Ross and A.J. Wrath. 2015. "Process Evaluation of an Early-Intervention Program for Mood and Anxiety Disorders Among Older Adolescents and Young Adults." Psychiatr Serv 66(10): 1113-7 doi: 10.1176/ appi. ps201400256

Otte, C. 2011. "Cognitive Behavioral Therapy in Anxiety Disorders: Current State of the Evidence.” Dialogues Clin Neurosci 13(4): 413-21. 
Perry, A., N. Tarrier, R. Morriss, E. McCarthy and K. Limb. 1999. "Randomised Controlled Trial of Efficacy of Teaching Patients With Bipolar Disorder to Identify Early Symptoms of Relapse and Obtain Treatment." BMJ 318(7177): 149-53.

Rapee, R.M., C.A. Schniering and J.L. Hudson. 2009. “Anxiety Disorders During Childhood and Adolescence: Origins and Treatment." Annu Rev Clin Psychol 5: 311-41.

Ross, E., E. Vingilis and E. Osuch. 2012. "An Engagement and Access Model for Healthcare Delivery to Adolescents With Mood and Anxiety Concerns." Early Interv Psychiatry 6(1): 97-105.

Smith, R.A., V. Cokkinides and H.J. Eyre. 2006. "American Cancer Society Guidelines for the Early Detection of Cancer, 2006." CA Cancer J Clin 56(1): 11-25; Quiz 49-50.

Snowdon, A. 2014. Graham Boeckh Foundation: Headspace Business Case. London, ON: International Centre for Health Innovation.

Vittengl, J.R., L.A. Clark, T.W. Dunn and R.B. Jarrett. 2007. "Reducing Relapse and Recurrence in Unipolar Depression: A Comparative Meta-Analysis of Cognitive-Behavioral Therapy's Effects." J Consult Clin Psychol 75(3): 475-88.

\section{About the Authors}

Elizabeth A. Osuch, M.D., University of Western Ontario, Schulich School of Medicine and Dentistry, Department of Psychiatry, London Health Sciences Centre; Lawson Health Research Institute, London, Ontario. Dr. Osuch can be reached by email at: Elizabeth.osuch@Ihsc.on.ca.

Evelyn Vingilis, Ph.D, University of Western Ontario, Schulich School of Medicine and Dentistry: Department of Family Medicine; The Western Centre for Public Health and Family Medicine

Sandra Fisman, M.D., University of Western Ontario, Schulich School of Medicine and Dentistry, Department of Psychiatry, London Health Sciences Centre; University of Western Ontario, Schulich School of Medicine and Dentistry, Division of Child and Adolescent Psychiatry, Departments of Psychiatry, Pediatrics and Family Medicine, London Health Sciences Centre

Carolyn Summerhurst, M.S.W., University of Western Ontario, Schulich School of Medicine and Dentistry, Department of Psychiatry, London Health Sciences Centre 